\title{
Zur Bestimmung präzipitierender Antikörper mit der umgekehrten einfachen radialen Immunodiffusion
}

\author{
Von A. Oberdorfer und H. Blaufuss \\ Institut für Klinische Chemie und Klinische Biochemie \\ (Direktor: Prof. Dr. A. Oberdorfer) der Technischen Universität München
}

(Eingegangen am 9. Juni 1972)

Bei der Bestimmung präzipitierender Antikörper mit der umgekehrten einfachen radialen Immunodiffusion und der quantitativen Immunopräzipitation ergab ein mit Kaninchenantiseren gegen Humanserumalbumin durchgeführter Verglcich dieser beiden Methoden folgende Resultate: Die umgekehrte einfache radiale Immunodiffusion ist spezifischer als die quantitative Immunopräzipitation. Die Empfindlichkeit der beiden Methoden ist annähernd gleich. Die Streuung der umgekehrten einfáchen radialen Immunodiffusion ist geringer als diejenige der quantitativen Immunopräzipitation. Die Korrelation zwischen der umgekehrten einfachen radialen Immunodiffusion und der quantitativen Immunopräzipitation ist hoch signifikant $(p<0,001)$. Hinsichtlich Arbeitszeit, Reaktionszeit, apparativer Ausrüstung und benötigter Probenmenge ist die umgekehrte einfache radiale Immunodiffusion weniger aufwendig als die quantitative Immunopräzipitation. Für ein Ergebnis der umgekehrten einfachen radialen Immunodiffusion kann mit Hilfe der Regressionsgleichung der entsprechende Wert der quantitativen Immunopräzipitation berechnet werden. Die gewählten Toleranzbereiche gewährleisten eine Sicherheitswahrscheinlichkeit von $95 \%$.

\section{Determination of precipitating antibodies by reversed single radial immunodiffusion}

The method of reversed single radial immunodiffusion for the determination of precipitating antibodies was compared with quantitative immunoprecipitation, using the system rabbit antiserum-human serum albumin. Reversed single radial immunodiffusion is more specific than quantitative immunoprecipitation. The sensitivities of the two methods are approximately the same. Reversed single radial immunodiffusion shows less variability than quantitative immunoprecipitation. The correlation betwcen reversed single radial immunodiffusion and quantitative immunoprecipitation is highly significant $(p<0.001)$. Concerning time of operation, reaction time, equipment and amount of sample required, the reversed single radial immunodiffusion is less demanding than quantitative immunoprecipitation. With the aid of the regression equation, the result from reversed single radial immunodiffusion can be used to calculate the corresponding value for the quantitative immunoprecipitation. The chosen tolerance range guarantees a confidence level of $95 \%$.

Die Bestimmung präzipitierender Antikörper mit der quantitativen Immunopräzipitation nach HEIDELBERGER $(1,2)$ ist bekanntlich mit einem relativ großen Zeit- und Arbeitsaufwand verbunden; außerdem werden hierbei verhältnismäßig große Mengen des zu untersuchenden Antiserums benötigt. Es fehlte daher nicht an Versuchen, die Originalmethode zu modifizieren oder durch andere Verfahren zu ersetzen. In letzter Zeit wurde auch die einfache radiale Immunodiffusion zur Bestimmung präzipitierender Antikörper herangezogen (3-7). Die besondere Eignung der „umgekehrten“" einfachen radialen Immunodiffusion zur Antikörper-Bestimmung wurde kürzlich durch die MRC Working Party on the Clinical Use of Immunological Reagents (8) hervorgehoben, die diese Methode als Testmethode zur Standardisierung von Antiseren empfahl. Im folgenden soll über Ergebnisse berichtet werden, die bei vergleichenden Untersuchungen der umgekehrten einfachen radialen Immunodiffusion und der quạtitativen Immunopräzipitation erhalten wurden.

\section{Material und Methoden}

Als Antigen wurde Humanserumalbumin (Humanalbumin trocken, reinst, der Firma Behringwerke AG, Marburg/Lahn) verwendet. Das bis zur Gewichtskonstanz bei $70^{\circ} \mathrm{C}$ über Phosphorpentoxid in Vakuum getrocknete Präparat hatte einen Stickstoffgehalt von
$15,9 \%$. Bei der Untersuchung in der analytischen Ultrazentrifuge (Spinco Modell E) wurde eine sedimentierende Komponente erhalten. Bei der Immunoelektrophorese mit polyvalentem Antihumanserum kam eine Präzipitationslinie zur Darstellung. Die Herstcllung der Antiseren erfolgte durch Immunisierung von Kaninchen.

Die quantitative Immunopräzipitation wurde in $0,05 \mathrm{~mol} / \mathrm{l}$ Natriumphosphatpuffer $\mathrm{pH} \mathrm{7,2} \mathrm{durchgeführt.} \mathrm{Hierzu} \mathrm{wurden} 0,5 \mathrm{ml}$ Antiserum und $0,5 \mathrm{ml}$ Antigenlösung gemischt. Für die Präzipitationskurve wurden 10 Ansätze mit Antigenkonzentrationen entsprechend $10-320 \mu \mathrm{g} \mathrm{N} / \mathrm{ml}$ hergestellt. Die Ansätze wurden zwei Stunden bei $37^{\circ} \mathrm{C}$ und fünf Tage bei $4^{\circ} \mathrm{C}$ stehengelassen. Die hierbei entstandenen Immunopräzipitate wurden nach zweimaligem Waschen mit je $4 \mathrm{ml} 0,05 \mathrm{~mol} / 1$ Natriumphosphatpuffer $\mathrm{pH} \mathrm{7,2}$ unter Zusatz von $1 \mathrm{ml}$ eines Gemisches von konz. $\mathrm{H}_{2} \mathrm{SO}_{4}$, gesätt. $\mathrm{K}_{2} \mathrm{SO}_{4}$-Lösung und Selen verascht. Die Wasserdampfdestillation wurde in "einer modifizierten PARNASS-WAGNER-Apparatur (9) durchgeführt. Das Ammoniak wurde in Borsäure aufgefangen und durch potentiometrische Endpunkttitration mit 0,05 $\mathrm{mol} / 1 \mathrm{HCl}$ bestimmt. Berechnung: $1 \mu 10,05 \mathrm{~mol} / 1 \mathrm{HCl}=0,7 \mu \mathrm{g} \mathrm{N}$. Die umgekebrte einfache radiale Immunodiffusion wurde in Agarosegel durchgeführt. Zur Herstellung der antigenhaltigen Agarosegele wurden $100 \mathrm{ml}$ einer 1,5proz. Agaroscpufferlösung (Agarose reinst, Serva Entwicklungslabor, Heidelberg, $0,05 \mathrm{~mol} / 1$ Natriumphosphatpuffer $\mathrm{pH} 7,2)$ bei $56^{\circ} \mathrm{C}$ mit $1 \mathrm{ml}$ Antigenlösung $(1 \mathrm{mg}$ bzw. $2 \mathrm{mg}$ bzw. $5 \mathrm{mg}$ Humanalbumin, gelöst in $0,05 \mathrm{~mol} / \mathrm{l} \mathrm{Na}$ triumphosphatpuffer pH 7,2) gemischt. Die auf diese Weise hergestellten Agarosepufferlösungen enthalten 0,01 bzw. 0,02 bzw. 0,05 mg Humanalbumin $/ \mathrm{ml}$. Je $8,5 \mathrm{ml}$ der antigenhaltigen Agarosepufferlösungen wurden mit vorgewärmten Pipetten in runde, auf $40^{\circ} \mathrm{C}$ temperierte Kunststoffschalen (lecre Partigen-Platten der 
Behringwerke AG, Marburg/Lahn) pipettiert. In die erstarrte, $1 \mathrm{~mm}$ hohe Gelschicht wurden pro Schale 12 Löcher gestanzt (Lochdurchmesser: $3 \mathrm{~mm}$, Abstand von Loch zu Loch: etwa 1,5 cm). In jedes Loch wurden je $5 \mu \mathrm{l}$ der zu untersuchenden Probe eingefüllt. Zur Herstellung einer ,feuchten Kammer" wurde in die gelfreie Mitte jeder Kunststoffschale ein mit Wasser getränkter Schwamm gelegt und die Schale mit einem Deckel verschlossen. Zur Diffusion wurden die Schalen $72 \mathrm{~h}$ bei Raumtemperatur stehengelassen. Wie in Vorversuchen festgestellt wurde, ist nach dieser Zeit die vollständige Ausbildung des Immunopräzipitates auch bei Seren mit hohen Antikörpertitern beendet. Der Durčhmesser des äußeren Präzipitatsringes wurde ohne Anfärbung des Präzipitates mit Hilfe eines binocularen Meßmikroskops (Gesamtskala: $10 \mathrm{~mm}$; 1 Teilstrich $=0,1 \mathrm{~mm}$ ) gemessen. Zur Archivierung wurden die Platten ungefärbt im Durchlicht photographiert. Das Ausmessen der Durchmesser der äußeren Präzipitatringe kann auch auf der photographischen Aufnahme erfolgen, da durch den Lochdurchmesser eine konstante Vergleichsgröße gegeben ist.

Für die Mehrzahl der untersuchten Proben eignet sịch eine Antigenkonzentration von $0,02 \mathrm{mg}$ Humanalbumin/ml Agarosegel. Wie die Untersuchung der Präzision der Methode ergab, weisen die Variationskoeffizienten der Präzipitatdurchmesser in einem Bereich von $4,0 \mathrm{~mm}$ bis $8,0 \mathrm{~mm}$ nur geringgradige Unterschiede auf; oberhalb eines Grenzwertes von $8,0 \mathrm{~mm}$ steigen die Variationskoeffizienten der Präzipitatdurchmesser jedoch an, vorwiegend wohl infolge der Unschärfe des Präzipitatrandes und der gegenseitigen Beeinflussung benachbarter Präzipitatringe. Daher wurden Meßergebnisse von Präzipitaten mit Durchmessern von mehr als $8,0 \mathrm{~mm}$ nicht verwertet. Für die Untersuchung von Seren mit hohem Antikörpergehalt wurde eine Antigenkonzentration von $0,05 \mathrm{mg}$ Humanalbumin/ml Agarosegel verwendet. Für Proben mit relativ niedrigem Antikörpertiter wurde zur Erzielung größerer und dadurch genauer meßbarer Präzipitatdurchmesser eine Antigenkonzentration von $0,01 \mathrm{mg}$ Humanalbumin $/ \mathrm{ml}$ Agarosegel gewählt.

Das bei der umgekehrten einfachen radialen Immunodiffusion erhaltene Präzipitat besteht aus drei konzentrischen Ringzonen, die sich durch Intensität und Breite unterscheiden (Abb. 1). In der inneren Ringzone, die sich in der Abbildung an den das Loch umgebenden Lichtreflex anschließt, ist kein Präzipitat erkennbar. Die Breite dieser inneren Ringzone ändert sich bei steigender Antiserumkonzentration nur geting. Die mittlere Ringzone enthält ein deutlich erkennbares Präzipitat. Die bei konstanter Antigenkonzentration und bei steigendem Antiserumtiter resultierende $\mathrm{Zu}$ nahme des Gesamtpräzipitates betrifft vorwiegend diese mittlere Ringzone. Die äußere Ringzone wird dargestellt durch ein scharf umschriebenes, relativ schmales Präzipitat starker Intensität. Die Breite und Intensität der äußeren Ringzone nimmt bei konstantem Antiserumtiter mit steigender Antigenkonzentration zu. Die Beziehung zwischen Präzipitatdurchmesser und Antikörpergehalt des Antiserums wird ausgedrückt durch die Gleichung

$$
\mathrm{S}=\mathrm{S}_{0}+\mathrm{K} \cdot \mathrm{C}_{\mathrm{AS}}
$$

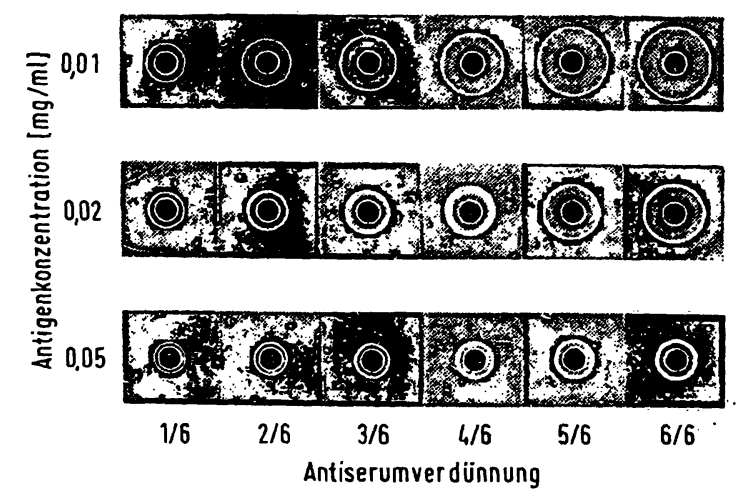

Abb. 1

Bestimmung präzipitierender Antikörper durch umgekehrte einfache radiale Immunodiffusion. Durchmesser der Immunopräzipitate in Abhängigkeit vom Antikörpergehalt der Antiseren (Antiserumverdün-
nung) und von der Antigenkonzentration

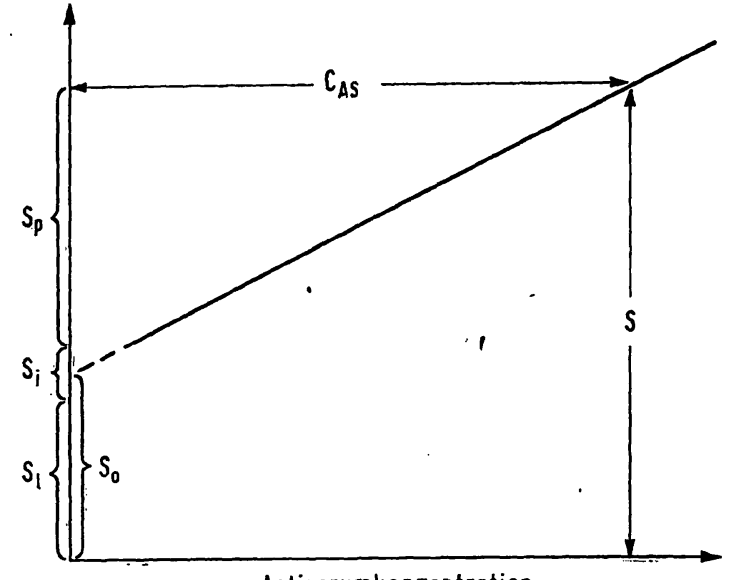

Antiserumkonzentration

Abb. 2

Bestimmung präzipitierender Antikörper durch̆ umgekehrte einfache radiale Immunodiff̧usion. Beziehung zwischen Präzipitatdurchmesser und Antiserumkonzentration. $\mathbf{S}=$ gemessener Durchmesser (sog. Präzipitatdurchmesser), $S_{l}=$ Durchmesser des Loches, $S_{1}=$ doppelte Breite der inneren Ringzone, $S_{p}=$ doppelte Breite der mittleren und
äußeren Ringzone, $S_{0}=$ Präzipitatdurchmesser bei der Antiserumäußeren $\begin{gathered}\text { Ringzone, } \\ \text { konzentration Null, } \mathrm{C}_{\mathrm{A}} \mathrm{S}=\text { Antiserumkonzentration }\end{gathered}$

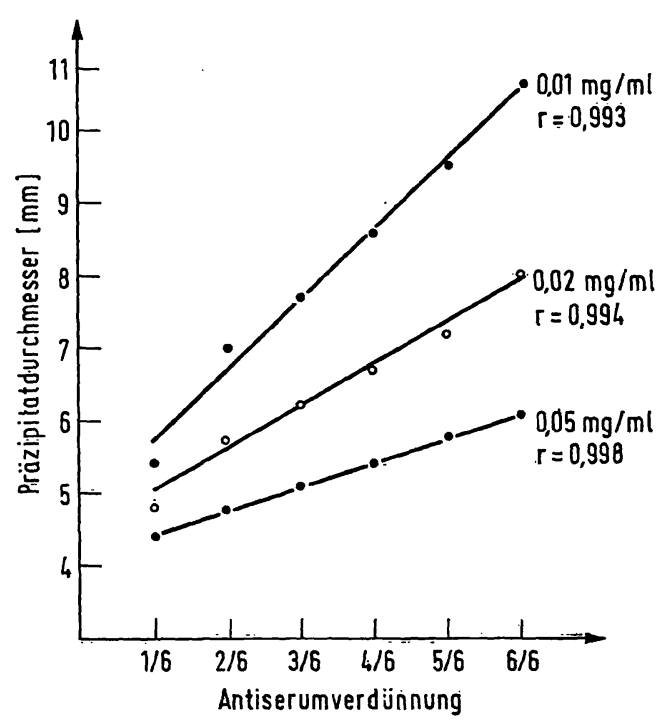

Abb. 3

Bestimmung präzipitierender Antikörper durch umgekehrte einfache radiale Immunodiffusion. Beziehung zwischen Präzipitatdurchmesser (Ordinate) und Antiserumkonzentration (Abszisse) bei 3 verschiedenen sind ausgedrückt durch die Antiserumverdünnung, $d . h$. däs Verhältnis des in den einzelnen Ansätzen enthaltenen Antiserumantéils zum Gesamtvolumen. $\mathrm{r}=$ Korrelationskoeffizient

$S$ ist der Durchmesser des äußeren Präzipitatringes, d: $h$. des äußeren Randes der äußeren Ringzone. S setzt sich aus dem Durchmesser des Loches und der doppelten Breite der drei Ringzonen zusammen. Im folgenden wird S als „Präzipitatdurchmesser“ bezeichnet.

$\mathrm{S}_{0}$ ist der Präzipitatdurchmesser bei der Antiserumkonzentration Null.

Der Faktor $\mathrm{K}$ drückt die Steigung einer Geraden aus, die man erhält, wenn man die Präzipitatdurchmesser verschiedener Konzentrationen eines Antiserums in ein Koordinatensystem einträgt (Abszisse: Antiserumkonzentration, Ordinate: Präzipitatdurchmesser). Der Faktor $\mathrm{K}$ ist abhängig von der Antigenkonzentration. Die Beziehung zwischen Faktor $\mathrm{K}$ und Antigenkonzentration ist umgekehrt proportional.

Mit $\mathrm{C}_{\mathrm{AS}}$ wird die Antiserumkonzentration bezeichnet (Abb. 2). Zur Prüfung det Linearität der Beziehung zwischen Präzipitatdurchmesser und Antiserumkonzentration wurde die Korrelation zwischen Präzipitatdurchmesser und Antiserumverdünnung bei verschiedenen Antigenkonzentrationen bestimmt (Abb. 3). Hierbei wurden hoch signifikante Korrelationen festgestellt ( $p<0,001)$. 


\section{Statistische Methodik}

Zur Bestimmung der Beziehung zwischen der quantitativen Immunopräzipitation und der umgekehrten einfachen radialen Immunodiffusion wurde der lineare Regressionsansatz benutzt. Als Maß für die Straffheit des linearen Zusammenhangs wurde der Korrelations-Koeffizient ( $r$ ) verwendet. -

Im Ansatz der Regressionsgleichung $\mathrm{y}=\mathrm{a}+\mathrm{bx}+\mathrm{e}$ wird die Streuung der Werte $y_{1}$ um die Regressionsgerade durch die Zufallsvariable e angedeutet. Die Zufallsvariable e wird als normalverteilt $\left[N\left(O, \sigma_{R}\right)\right]$ vorausgesetzt. Als Schätzung für $\sigma_{R}$, der Streuung der Einzelpunkte um die Regressionsgerade, wurde die Formel

$$
s_{R}=\sqrt{\frac{1}{n-1} \sum_{i=1}^{1}\left(y_{i}-y_{1 R}\right)^{2}}
$$

benutzt. Aus der Regressionsgleichung läßt sich für jeden Wert $\mathbf{x}$ der Erwartungswert der Zufallsvariablen y berechnen. Dieser wird mit $y_{R}$ bezeichnet. Der tatsächliche Wert von y streut um diesen mittleren Wert. Es wurde deshalb der Toleranzbereich nach folgender Formel bestimmt:

$$
\mathrm{y}=\mathrm{y}_{\mathrm{R}} \pm \mathrm{ts}
$$

Der Toleranzfaktor ( $t$ ) wurde dem Tabellenwerk von OwEN (10) entnommen. Er ist abhängig von der Anzahl der durchgeführten Bestimmungen und von den Sicherheitswahrscheinlichkeiten (jeweils 95\%). Diese sagen aus, daß mit 95prozentiger Wahrscheinlichkeit auch in späteren Stichproben $95 \%$ aller Werte im angegebenen Bereich liegen. Bei dieser Berechnung wird der im Rahmen des Stichprobenfehlers von Stichprobe zu Stichprobe etwas abweichende Verlauf der Schätzkurve in der Angabe des Toleranzbereiches mit berücksichtigt.

Die Rechenarbeiten wurden mit dem Tischcomputer Programma 101 der Firma Olivetti und der Rechenmaschine Combitron S der Firma Diehl durchgeführt.

\section{Ergebnisse und Diskussion}

Die Untersuchung der umgekehrten einfachen radialen Immunodiffusion und der Vergleich dieser Methode mit der quantitativen. Immunopräzipitation wurden nach folgenden Kriterien durchgeführt: Spezifität, Empfindlichkeit, Präzision, Richtigkeit, zeitlicher Arbeitsaufwand, Reaktionszeit, apparativer Aufwand, benötigte Mengen an Untersuchungsmaterial.
Zur Bestimmung der Spezifität der Methoden wurden Seren von nichtimmunisierten Kaninchen untersucht. Mit der quantitativen Immunopräzipitation wurde hierbei pro Ansatz $(n=20)$ ein Stickstoffgehalt von $28 \pm 4$ $\mu \mathrm{g} N$ erhalten. Dieser Stickstoff besteht aus dem Stickstoff von auch nach zweimaligem Waschen noch am Glas haftenden Serumresten und dem Stickstoff der Reagenzien. Der Anteil des Reagenzien-Stickstoffes beträgt $17 \pm 2 \mu \mathrm{g}$ N. Mit der umgekehrten einfachen radialen Immunodiffusion konnte bei der Untersuchung von Seren nichtimmunisierter Tiere kein meßbares Präzipitat festgestellt werden. Die umgekehrte einfache radiale Immunodiffusion erwies sich demnach im Vergleich zur quantitativen Immunopräzipitation als die spezifischere Methode.

Als Kriterium für die Empfindlichlecit wurde die untere Nachweisgrenze gewählt. Diese kann durch Mittelwert plus drei Standardabweichungen des Blindwertes $(\bar{x}+3 s)$ definiert werden (11). Wenn man den mit der quantitativen Immunopräzipitation bei der Untersuchung von Seren nichtimmunisierter Kaninchen erhaltenen Stickstoffwert als Blindwert betrachtet, ergibt sich für diese Methode eine untere Nachweisgrenze von $40 \mu \mathrm{g} \mathrm{N}(\overline{\mathrm{x}}=28 \mu \mathrm{g} \mathrm{N}, \mathrm{s}=4 \mu \mathrm{g} \mathrm{N})$. Die obengenannte Definition ist bei der umgekehrten einfachen radialen Immunodiffusion zur Beschreibung der unteren Nachweisgrenze nicht anwendbar, da bei dieser Methode kein meßbarer Blindwert erhalten wird. An Stelle des Blindwertes kann man zur Bestimmung der unteren Nachweisgrenze den kleinsten noch sicher meßbaren Immunopräzipitatdurchmesser heranziehen, da dessen Streuung ähnlich der Streuung des vermuteten Blindwertes ist. Untersucht man die Streuung eines der Antiseren, dessen Antikörpergehalt in der Nähe der unteren Nachweisgrenze liegt, so erhält man bei einem Mittelwert $\bar{x}=4,0333 \mathrm{~mm}$ eine Standardabweichung von $\mathrm{s}=0,0322 \mathrm{~mm}(\mathrm{n}=24)$. Die untere Nachweisgrenze

Tab. 1

Bestimmung präzipitierender Antikörper durch umgekehrte einfache radiale Immunodiffusion (UERID) und quantitative Immunopräzipitation (IP): Vergleich der unteren Nachweisgrenzen beider Methoden durch Untersuchung von Verdünnungsreihen ${ }^{\text {) }}$ von 4 verschiedenen Antiseren (I, II, III, IV)

\begin{tabular}{|c|c|c|c|c|c|c|c|c|}
\hline \multirow{2}{*}{$\begin{array}{l}\text { Antiserum- } \\
\text { verdünnung }\end{array}$} & \multicolumn{2}{|c|}{ Antiserum I } & \multicolumn{2}{|c|}{ Antiserum II } & \multicolumn{2}{|c|}{ Antiserum III } & \multicolumn{2}{|c|}{ Antiserum IV } \\
\hline & $\begin{array}{l}\text { UERID } \\
\text { (mm) }\end{array}$ & $\begin{array}{c}\text { IP } \\
(\mu \mathrm{g} \mathrm{N})\end{array}$ & $\begin{array}{l}\text { UERID } \\
\text { (mm) }\end{array}$ & $\begin{array}{c}\text { IP } \\
(\mu \mathrm{g} \mathrm{N})\end{array}$ & $\begin{array}{l}\text { UERID } \\
(\mathrm{mm})\end{array}$ & $\begin{array}{c}\text { IP } \\
(\mu \mathrm{g} \mathrm{N})\end{array}$ & $\begin{array}{l}\text { UERID } \\
(\mathrm{mm})\end{array}$ & $\begin{array}{c}\text { IP } \\
(\mu \mathrm{g} \mathrm{N})\end{array}$ \\
\hline $1: 2$ & nd & nd & nd & nd & 7,0 & 215 & nd & nd \\
\hline $1: 5$ & nd & nd & nd & nd & 5,1 & 98 & nd & nd \\
\hline $1: 10$ & 4,5 & 81 & 5,8 & 127 & 4,4 & 53 & 5,0 & 87 \\
\hline $1: 20$ & 4,2 & 54 & 4,9 & 78 & 4,2 & 42 & 4,5 & 58 \\
\hline $1: 30$ & 4,1 & 41 & 4,5 & 60 & 4,1 & 37 & 4,2 & 49 \\
\hline $1: 40$ & 4,0 & 37 & 4,4 & 58 & vnm & 29 & 4,1 & 42 \\
\hline $1: 50$ & 4,0 & 35 & 4,3 & 46 & nv & 29 & 4,0 & 36 \\
\hline $1: 60$ & vnm & 34 & 4,3 & 41 & nv & 34 & 4,0 & 35 \\
\hline $1: 70$ & vnm & 34 & 4,2 & 36 & . nv & 32 & 4,0 & 35 \\
\hline $1: 80$ & nv & 35 & 4,2 & 39 & nd & nd & nv & 38 \\
\hline $1: 90$ & nd & nd & 4,1 & 38 & nd & nd & nv & 35 \\
\hline $1: 100$ & nd & nd & 4,0 & 36 & nd & nd & nd & nd \\
\hline
\end{tabular}

nd $=$ Untersuchung wurde nicht durchgeführt $\quad$ vnm $=$ Präzipitatring vorhanden, aber nicht meßbar nv $=$ Präzipitatring nicht vorhanden

1) Das zur Verdünnung der Antiseren verwendete Mischserum aus Seren von 9 nichtimmunisierten Kaninchen ergab mit der quantitativen I mmunopräzipitation $30 \mu \mathrm{g}$. Bei der Untersuchung dieses Mischserums mit der umgekehrten einfachen radialen Immunodiffusion wurde kein meßbares Präzipitat festgestellt. 
würde demnach $4,1299 \mathrm{~mm}$ betragen. Berechnet man für einen Präzipitatdurchmesser von $4,0 \mathrm{~mm}$ den entsprechenden Wert der quantitativen Immunopräzipitation, so erhält man bei der Antigenkonzentration von $0,01 \mathrm{mg}$ Humanalbumin/ml Agarosegel hierfür einen Wert von $40 \mu \mathrm{g} \mathrm{N}$. Dieser Wert entspricht der unteren Nachweisgrenze der quantitativen Immunopräzipitation. Ein Unterschied zwischen den unteren Nachweisgrenzen der quantitativen Immunopräzipitation und der umgekehrten einfachen radialen Immunodiffusion ist demnach nicht zu erkennen. Zum Vergleich der unteren Nachweisgrenzen beider Methoden wurden außerdem Verdünnungsreihen von Antiseren untersucht. Wie aus Tabelle 1 ersichtlich ist, konnten auch hierbei keine Unterschiede der Empfindlichkeit beider Methoden festgestellt werden.

Zur Untersuchung der Präzision wưrde für beide Methoden die Streuung in der Serie bestimmt. Die hierbei gewonnenen Ergebnisse sind in den Tabellen 2 und 3 enthalten. Bei der quantitativen Immunopräzipitation ist die Streuung in der Serie abhängig vom Antikörpergehalt der Antiseren bzw. von der Menge des gemessenen Präzipitatstickstoffes. Mit steigendem Präzipitatstickstoff sinkt der Variationskoeffizient von 5,8\% auf 1,5\%. Bei der umgekehrten einfachen radialen Immunodiffusion ist eine derartige Abhängigkeit der Streuung der Methode von der Antikörperkonzentration des Antiserums nicht zu erkennen. Der Variations-Koeffizient der Streuung in der Serie liegt bei der umgekehrten einfachen radialen Immunodiffusion $z$ wischen $0,8 \%$ und 1,6\%. Außerdem wurde bei dieser Methode auch die Streuung von Platte zu Platte bestimmt; hierbei wurde ein Variationskoeffizient von 2,5\% erhalten. Ein Vergleich der mit beiden Methoden bei der Untersuchung von Antiseren gleichhoher Antikörpergehalte gewonnenen Ergebnisse zeigt, daß insbesondere bei Antiseren mit niedrigem Antikörpergehalt die Streuung der quantitativen Immunopräzipitation größer ist als diejenige der umgekehrten einfachen radialen Immunodiffusion. Die Präzision der umgekehrten einfachen radialen Immunodiffusion ist demnach bei der Untersuchung von Antiseren mit niedrigem Antikörpergehalt besser als diejenige der quantitativen Immunopräzipitation.

Tab. 2

Bestimmung präzipitierender Antikörper durch quantitative Immunopräzipitation: Streuung in der Serie; $s$ und VK resultieren aus einer Aufrundung von Zahlen mit vier Kommastellen

\begin{tabular}{rccc}
\hline $\begin{array}{c}\overline{\mathrm{x}} \\
(\mu \mathrm{g} \mathrm{N})\end{array}$ & $\begin{array}{c}\mathrm{s} \\
(\mu \mathrm{g} \mathrm{N})\end{array}$ & $\begin{array}{c}\text { VK } \\
(\%)\end{array}$ & $\mathrm{n}$ \\
\hline 61 & 3,0 & 4,9 & 23 \\
82 & 4,7 & 5,8 & 24 \\
102 & 3,3 & 3,2 & 24 \\
156 & 4,0 & 2,6 & 23 \\
160 & 6,2 & 3,9 & 24 \\
167 & 4,2 & 2,5 & 24 \\
338 & 6,9 & 2,0 & 24 \\
390 & 7,7 & 2,0 & 23 \\
429 & 9,8 & 2,3 & 24 \\
565 & 12,9 & 2,3 & 24 \\
759 & 14,5 & 1,9 & 24 \\
1115 & 16,9 & 1,5 & 23 \\
\hline
\end{tabular}

Tab. 3

Bestimmung präzipitierender Antikörper durch umgekehrte einfache radiale Immunodiffusion: Streuung in der Serie; $s$ und VK resultieren aus einer Aufrundung von Zahlen mit vier Kommastellen

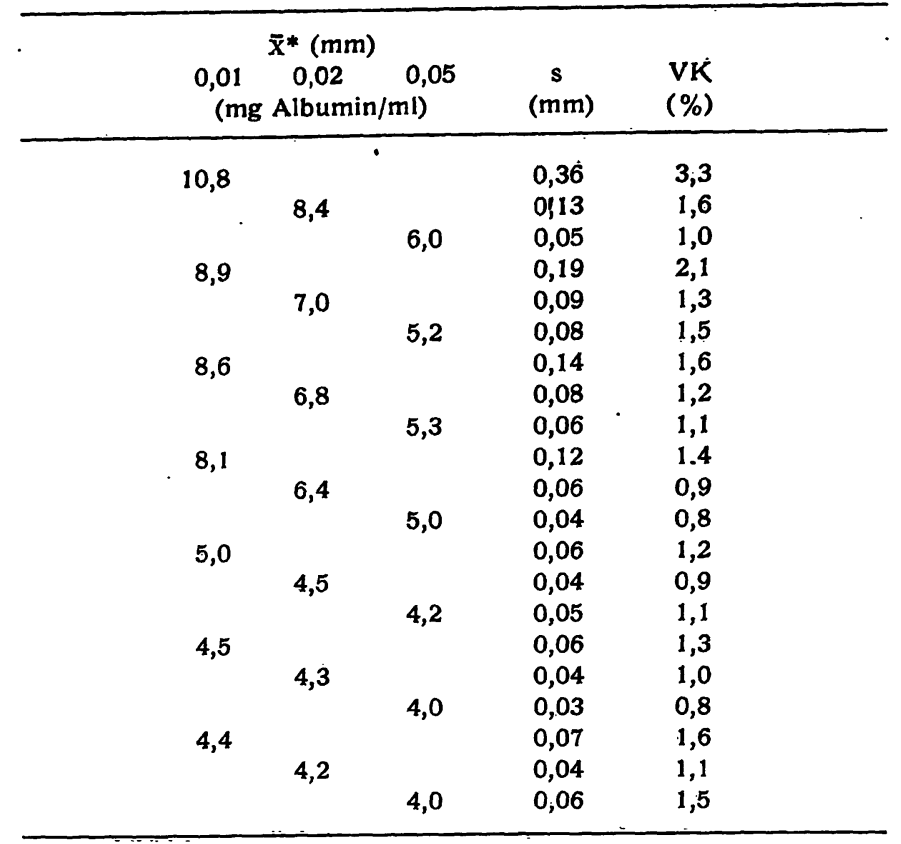

$* n=24$, bestimmt in zwei Diffusionsplatten.

Zur Prüfung der Ricbtigkeit einer Analysenmethode stehen bekanntlich folgende Verfahren zur Verfügung: Untersuchung von Proben mit bekanntem Gehalt, Wiederfindungsversuche, Vergleich mit einer anderen, bereits bewährten Methode. Die beiden erstgenannten Verfahren können in vorliegendem Fall nicht angewandt werden, da weder standardisierte Antiseren noch entsprechende Antikörperpräparate zur Verfügung stehen. Zur Prüfung der Richtigkeit der umgekehrten einfachen radialen Immunodiffusion wurde deshalb ein Vergleich mit der quantitativen Immunopräzipitation durchgeführt. Die Untersuchung der Korrelation zwischen diesen beiden Methoden erfolgte bei drei verschiedenen Antigenkonzentrationen. Hierbei wurden die Korrelationskoeffizienten $0,9040,0,9420$ und 0,9350 erhalten (Tab. 4, Abb. 4). Die Irrtumswahrscheinlichkeit ist kleiner als $0,1 \%$. Die Korrelation zwischen umgekehrter einfacher radialer Immunodiffusion und quantitativer Immunopräzipitation ist demnach hoch signifikant. Es ist deshalb möglich, mit Hilfe der in Tabelle 4 angegebenen linearen Regressionsgleichung unter Berücksichtigung eines Toleranzbereiches für ein Ergebnis der umgekehrten einfachen radialen Immunodiffusion den entsprechenden Wert $(\mu \mathrm{g} N)$ der quantitativen Immunopräzipitation zu berechnen. Wie aus Abbildung $4 \mathrm{zu}$ erkennen ist, nimmt die Streuung der Einzelpunkte um die Regressionsgerade in Abhängigkeit von der Größe des Meßwertes, d. h. mit steigendem Antikörpergehalt, zu. Wählt man jedoch - wie hier vorgeschlagen einen Toleranzbereich von 95proz. Sicherheit, so ist darin auch die Streuung größerer Meßwerte enthalten. 
Tab. 4

Korrelation zwischen umgekehrter einfacher radialer Immunodiffusion (UERID) und quantitativer Immunopräzipitation

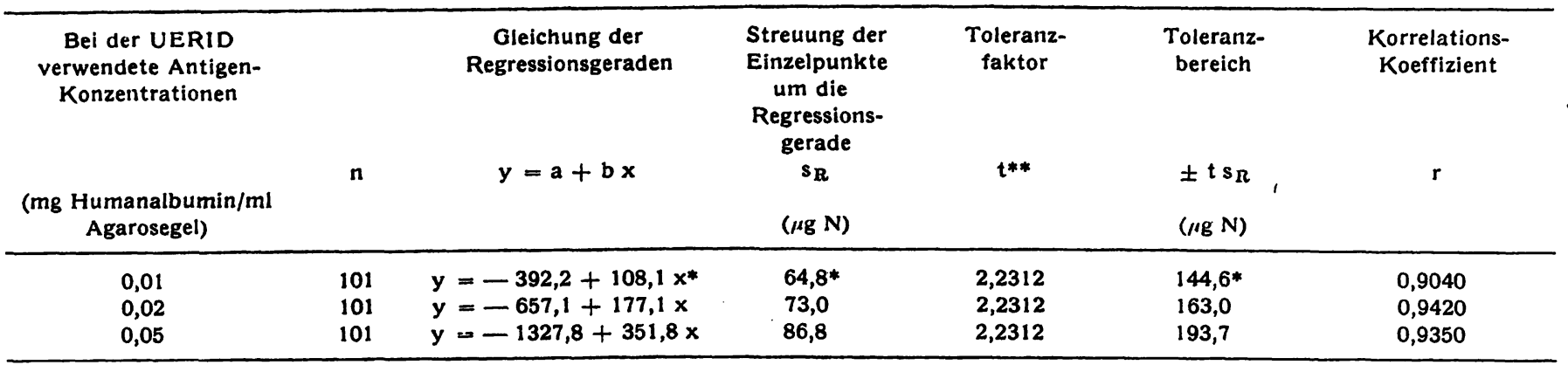

* Die Zahlen dieser Kolonnen resultieren aus einer Aufrundung von Zahlen mit vier Kommastellen.

** Die Werte für $t$ wurden dem Tabellenwerk von OWEN (10) entnommen.

Abb. 4

Korrelation zwischen umgekehrter einfacher radialer Immunodiffusion und quantitativer Immunopräzipitation bei 3 versionsgeraden mit Korrelationskoeffizienten s. Tab. 4 schiedenen Antigenkonzentrationen. Gleichungen der Regres-
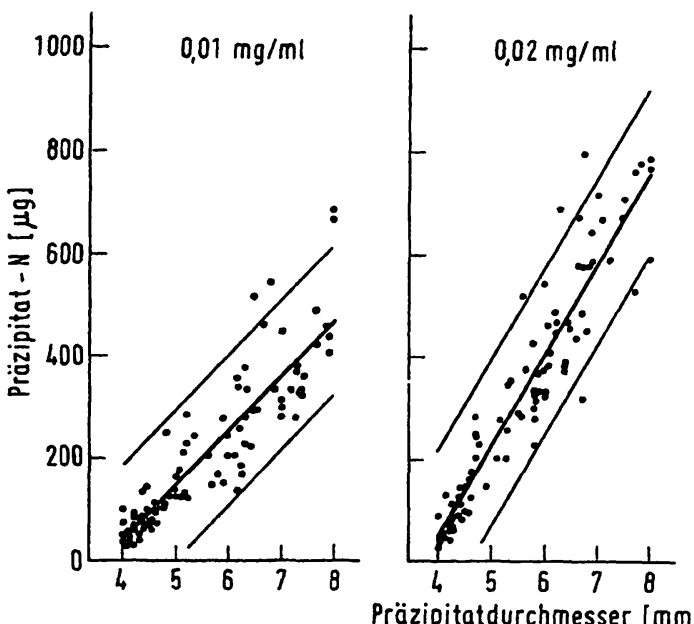

Präzipitatdurchmesser [mm]

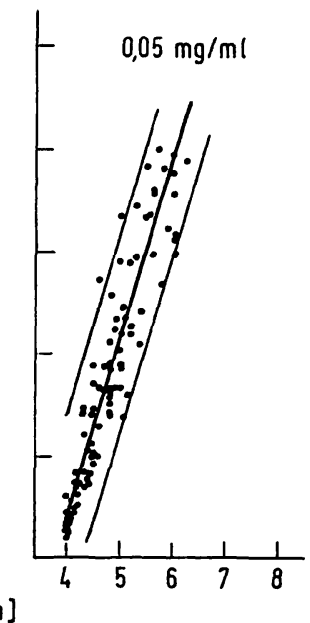

Tab. 5

Bestimmung präzipitierender Antikörper mit der umgekehrten einfachen radialen Immunodiffusion und der quantitativen Immunopräzipitation. Vergleich des bei der Untersuchung von jeweils 120 Proben erforderlichen Zeitaufwandes

dadurch beeinträchtigt werden, $\mathrm{da} ß$ verschieden große Antikörpermoleküle unterschiedlich weit in das Gel hineindiffundieren. Der Präzipitatdurchmesser kann demnach auch von der Zusammensetzung des Antikörpergemisches im Antiserum abhängen. Diese Überlegungen können dann an Bedeutung gewinnen, wenn die Methode zur Untersuchung des Verlaufs der Antikörperbildung angewendet wird. $\mathrm{Ob}$ und in welchem Ausmaß sich dieser Störfaktor auf die Ergebnisse der umgekehrten einfachen radialen Immunodiffusion tatsächlich auswirkt, wurde im Rahmen dieser Arbeit jedoch nicht untersucht.

Die für die Durchführung der umgekehrten einfachen radialen Immunodiffusion und der quantitativen Immunopräzipitation benötigten Arbeitszeiten verhalten sich bei rationallem Ansatz großer Serien annähernd wie 1:16 (Tab. 5). Hierbei wurde nur die reine Arbeitszeit berücksichtigt, nicht dagegen die zwischen den einzelnen Arbeitsgängẹn liegenden Reaktionszeiten, wie z. B. Diffusionszeit, Immunopräzipitationszeit und Veraschungszeit.

Bei der umgekehrten einfachen radialen Immunodiffusion ist die Reaktionszeit auf die Diffusionszeit beschränkt, d. h. auf den Zeitabschnitt vom Einfüllen der Probe bis zur Messung des Präzipitationsdurchmessers (72 h). Bei der quantitativen Immunopräzipitation besteht die

\begin{tabular}{|c|c|c|c|}
\hline \multicolumn{2}{|c|}{$\begin{array}{l}\text { umgekehrte einfache radiale } \\
\text { Immunodiffusion }\end{array}$} & \multicolumn{2}{|c|}{$\begin{array}{c}\text { quantitative } \\
\text { Immunopräzipitation }\end{array}$} \\
\hline Teilschritte & $\begin{array}{c}\text { Arbeitszeit } \\
\text { (h) }\end{array}$ & Teilschritte & $\begin{array}{l}\text { Arbeitszeit } \\
\text { (h) }\end{array}$ \\
\hline \multirow{7}{*}{$\begin{array}{c}\text { Herstellen von } \\
30 \text { Platten (3 Antigen- } \\
\text { konzentrationen) } \\
\text { Einfüllen der } \\
\text { Proben } \\
\text { Messen der Präzi- } \\
\text { pitatdurchmesser }\end{array}$} & \multicolumn{3}{|c|}{ Ansatz } \\
\hline & \multirow{3}{*}{4} & (120 Proben $=$ & \\
\hline & & 1200 Ansätze) & 14 \\
\hline & & Zentrifugieren & \\
\hline & \multirow[t]{2}{*}{2} & und Waschen & 14 \\
\hline & & Veraschen & 4 \\
\hline & \multirow[t]{2}{*}{2} & Destillieren & 48 \\
\hline & & Titrieren & 48 \\
\hline \multirow[t]{2}{*}{ Gesamte Arbeitszeit } & \multirow[t]{2}{*}{8} & Gesamte & \\
\hline & & Arbeitszeit & 128 \\
\hline
\end{tabular}

Reaktionszeit aus der Zeit zwischen Ansatz und Abzentrifugation des Immunopräzipitates $(120 \mathrm{~h})$ und der zur Veraschung benötigten Zeit (je nach Menge des zu veraschenden Präzipitates: $24-48 \mathrm{~h}$ ). Die quantitative Immunopräzipitation hat demnach eine annähernd zweimal so lange Reaktionszeit wie die umgekehrte einfache radiale Immunodiffusion. Der Mindestzeitaufwand (Arbeitszeit und Reaktionszeit) für eine Einzelbestimmung beträgt bei der umgekehrten einfachen radialen Immunodiffusion 3 Tage, bei der quantitativen Immunopräzipitation 7 Tage. 
Der apparative Aufivand für die umgekehrte einfache radiale Immunodiffusion beschränkt sich auf Heizplatten (zum Erhitzen der Agaroselösung und zum Vorwärmen der Kunststoffschalen) und auf ein Meßmikroskop zur Messung der Präzipitatdurchmesser. Für die Durchführung der quantitativen Immunopräzipitation benötigt man Einrichtungen zur Konstanthaltung der Temperatur, Zentrifugen zum Abzentrifugieren und Waschen des Immunopräzipitates, eine Veraschungseinrichtung, eine Anlage zur Wasserdampfdestillation sowie ein Gerät zur potentiometrischen Endpunkttitration.

Die benötigten Mengen an Untersuchungsmaterial sind für beide Methoden unterschiedlich. Für die Durchführung der umgekehrten einfachen radialen Immunodiffusion ist für einen Ansatz mit 3 verschiedenen Antigenkonzentrationen eine Probenmenge von insgesamt $15 \mu \mathrm{l}$ er- forderlich. Im Gegensatz dazu werden bei der quantitativen Immunopräzipitation für einen Ansatz mit 10 verschiedenen Antigenkonzentrationen $5 \mathrm{ml}$ Antiserum benötigt.

\section{Danksagung}

Herm Prof. Dr. H.-J. LANGe (Institut für Medizinische Statistik, Dokumentation und Datenverarbeitung der Techn. Universität München), Herrn Dr. N. Vrçror (Gesellschaft für Strahlenforschung $\mathrm{mbH}$, München, Forschurigsgruppe Med. Datenverarbeitung) sowie Herrn Priv.-Doz. Dr. Dr. D. Stam (Abteilung für Klinische Chemie am Max-Planck-Institut für Psychiatrie, München) danken wir für wertvolle Diskussionen und Beratungen in statistischen Fragen.

Fräulein H. MǘLLER danken wir für hervorragende technische Mitarbeit. Frau LANGENEGGer danken wir für Mịthilfe bei den Tierversuchen.

\section{Literatur}

1. Heidelberger, M., F. E. Kendall und C. M. Soo Hoo; J. exper. Med. 58, 137 (1933). - 2. Heidelberger, M. und F. E. Kendall, J. exper. Med. 62, 657 (1935). - 3. Stiehm, E. R., J. Laborat. Clin. Med., S. Louis 70, 528 (1967). - 4. VAERMAN, J.-P., A.-M. LEBace-Verheyden, L. Scolari und J. F. Heremans, Immunochemistry 6, 287 (1969). - 5. BECKER, W., Immunochemistry 6, 539 (1969). - 6. CowAN, K. M. und G. G. WAGNER, J. Immunol., Baltimore 105, 557 (1970). - 7. ReIMER, C. B.,
D. J. Phillips, S. E. Maddison und S. L. Shore, J. Laborat. Clin. Med., S. Louis 76, 949 (1970). - 8. MRC Working Party on the Clinical Use of Immunological Reagents; Immunology 20, 3 (1971). - 9. Markham, R., Biochem. J. 36, 790 (1942). - 10. OWEN, D. B., Handbook of statistical tables. Addison-Wesley Publ. Co. Palo Alto and London (1962). - 11. KaISER, H., Z. analyt. Chem. 209, 1 (1965).

Prof. Dr. A. Oberdorfer

Institut für Klin. Chemie u. Klin.

Biochemie der Techn. Universität München 8 München 80

Ismaninger Str. 22

Deutschland 


\section{W Walter de Gruyter Berlin-New York}

\section{Proceedings on the \\ International Research \\ Conference of \\ Proteinase Inhibitors}

Munich, November 4-6, 1970

Edited by $\mathrm{H}$. Fritz and $\mathrm{H}$. Tschesche

XII, 304 apges. With 246 figures and 95 tables. 1971. Bound DM 108, -

\section{Contents:}

Proteinase Inhibitors in Human Medicine - Specific Isolation Methods - Inhibition Mechanisme: Theories and Methods - Inhibitors of Plant Tissues - Basic Bovine Inhibitor and Related Inhibitors .

Pancreatic Secretory Inhibitors - Seminal Inhibitors, Inhibitors and Fertilization - Inhibitors from Dog Sudmandibular Glands, Ascaris Lubricoides and Leeches.

\section{Contributions by:}

Birk, Coan, Čechová, Dlouhá-Keil, Feeney, Feinstein, Fink, Fritż, Greene, Haendle, Heimburger, Hochstraßer, Huber, Ikenaka, Kassell, Laskowski Jr., Laskowski Sr., Liener, Peanasky, Rigbi, Ryan,

Schumacher, Shotton, Stevens, Tschesche, Werle, Zaneveld.

For USA and Canada:

Please send all orders to Walter de Gruyter Inc. 162 Firth Avenue, New York, N.Y. 10010

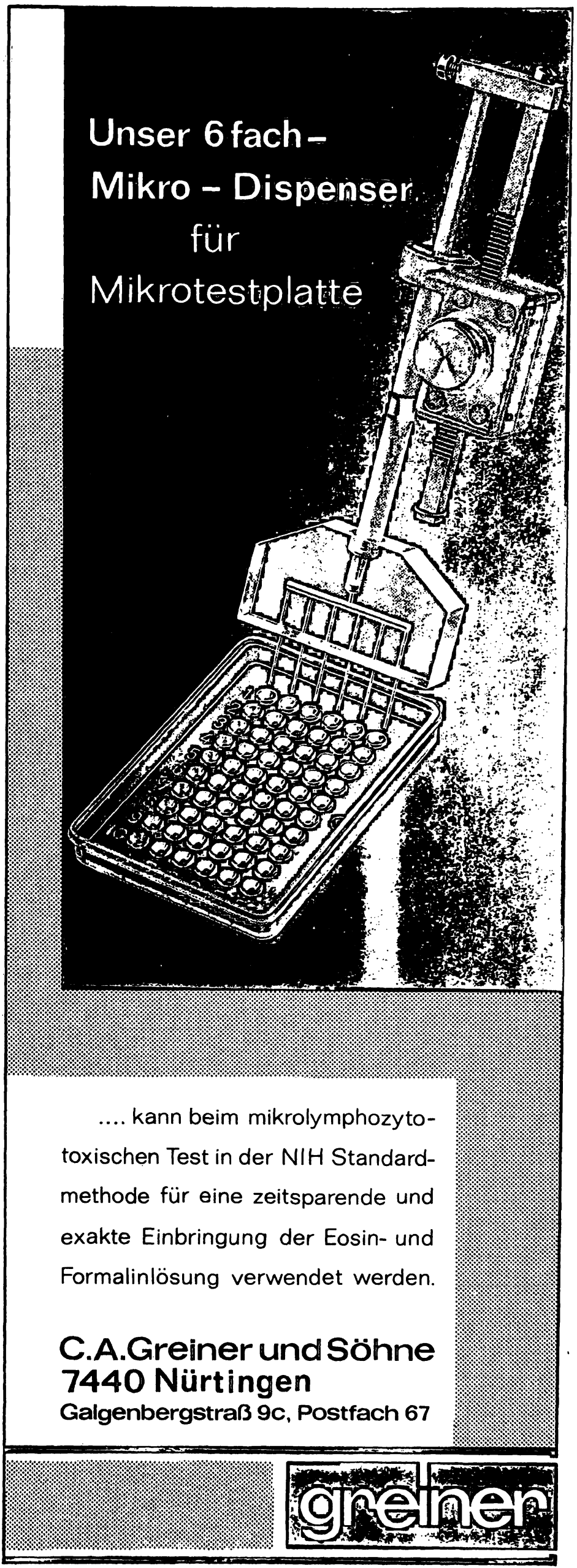




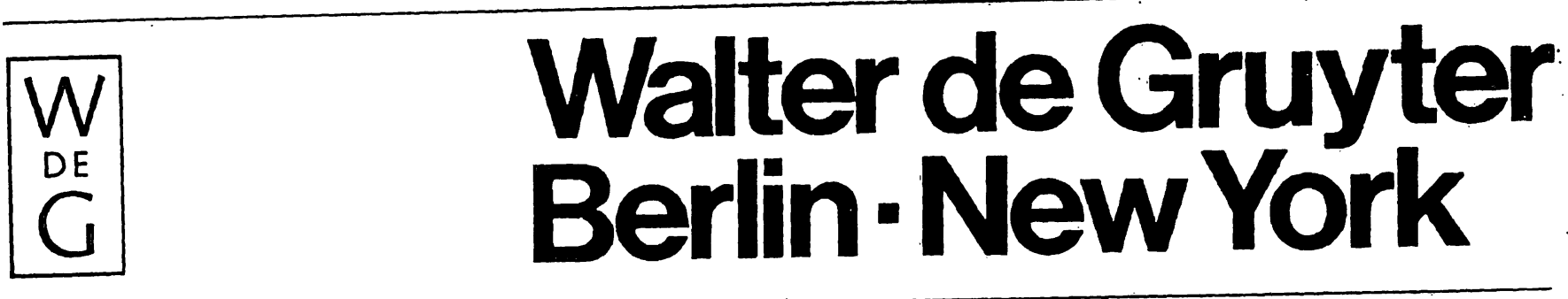

\section{Clinical Biochemistry}

\section{Principles and Methods}

Edited by H.-CH. CURTIUS

Medizinisch-Chemische Abteilung der

Universitäts-Kinderklinik, Zürich

and

MARC ROTH

Laboratoire Central

Hopital Cantonal universitaire, Geneve

61 authors from 11 different countries have contributed to this book which presents many of the techniques of interest to clinical chemists and clinical biochemists.

Current procedures are critically discussed, and special emphasis is given to new methods likely to become important in the coming years. A number of techniques are given in detail, and the others are presented with the appropriate references.

The book contains numerous tables and illustration, and an extensive index permits ready access to specific items.

Approx. 900 pages with many graphs

Size: $17 \times 24 \mathrm{~cm}$

Approx. DM 175, - / $\$ 55.50$

Prospective date of publication beginning of 1973

\section{Contents}

\section{General Methodology}

I. Preparation of Samples

(M. Roth and H.-Ch. Curtius)

II. Methods of Separation

Chromatography: Thin-Layer (H. K. Mangold) - Gel (H. Determann) Ion Exchange ( $H$. Determann) Gas-Liquid Chromatography and its Combination with other Analytical Techniques (H.-Ch. Curtius and J. A. Völlmin).

Electrophoresis: Membrane, incl. Immunoelectrophoresis, Gel (Agar, Starch, Polyacrylamide), Thin-Layer, High Voltage and Electro Focussing

(J. Jákó and W. Hitzig).

Ultracentrifugation $(\mathrm{H}$. Sund and F. Hucho). - Dialysis and Ultrafiltration (T. P. King). - Extraction by Adsorption (A. Niederwieser).

III. Methods of Analysis

Photometry: Absorption and Fluorimetry (M. Roth) - Flame Photometry (J. Frei) Atomic Absorption (H. Brandenberger). Isotope Techniques: Principles and Applications (H. J. Degenhart) Activation Analysis (D. Comar) Radioimmunoassays (J.-P. Felber) Protein-Binding Methods (A. Vermeulen). Mass Spectrometry (J. A. Völlmin). Electrochemical Methods (J. Bierens de Haan) - Ion-Selective Electrodes (J. T. Clerc, E. Pretsch). Calorimetric Methods (K. Levin). Enzymatic Analysis (M. Roth and J.-P. Felber). - Automation and Data Processing (J. Bierens de Haan) including GeMSAEC (N. G. Anderson). Ultramicro Techniques (M. Roth). Statistics (Th. Marthaler).-Quality Control and Normal Values (M. J. R. Healy and F. L. Mitchell).

\section{Detailed Procedures According to Groups of Substances}

IV. Hormones

Peptide Hormones (J.-P. Felber) Function Tests (R. Illig). - Steroid Hormones: Androgens, Progesterone and Metabolites, Corticosteroids (J.A. Völlmin, H.-Ch. Curtius, H. J. Degenhart and A. Vermeulen) - Estrogens ( $H$. Brever and L. Nocke-Finck) - Aldosterone (A. Aakvaag) - Function Tests (M. Zachmann). - Biologically Active Amines (C. Bohuon). - Thyroid Hormones: Protein-bound lodine (A. Uettwiler) Application of Radioactive lodine in Diagnosis and Treatment of Thyroid Diseases (W. D. Reitsma and M. G. Woldring).

V. Bile Acids (D. Mayer).
VI. Carbohydrates and Related Compounds.

Gas-Liquid Chromatography of Carbohydrates (W. W. Wells). - Glucose, Galactose, Fructose, Inulin and Ketone Bodies in Blood and Urine (E. Hultman). - Glycosaminoglycans (H. Greiling).

VII. Vitamins

Vitamin A (G. B. Brubacher and J. P. Vuilleumier) - Vitamin $B_{12}$ (U. G. Woldring and A. K. van Zanten) Vitamin C (G. B. Brubacher and J. P. Vuilleumier) - Vitamin D (E. Kodicek and D. E. M. Lawson). VIII. Lipids (H. K. Mangold) IX. Amino Acids (Th. Gerritsen and A. Niederwieser) - Fluorimetric Assay of Amino Acids (M. Roth).

$X$. Other Nitrogenous Compounds (G. Ceriotti).

XI. Enzymes

in Plasma (M. Roth, C. Bohuoñ, J. King and G. Ceriotti) - in Erythrocytes (W. Schröter) - in Leucocytes (J. Frei) Glycogen and Enzymes of Glycogen Metabolism (F. Huijing) - Enzymes of Fructose and Galactose Metabolism, Galactose-I-Phosphate (R. Gitzelmann) in Pancreatic and Duodenal Juice and in Feces (B. Borgström) in Intestinal Mucosa (S. Auricchio and A. Rubino) - Defects in Lipidoses and and their Prenatal Detection (R. O. Brady) - UDP-Glucuronyl-Transferase

(J. Frei) - Survey of Enzyme Assay Related to Inborn Errors of Metabolism (R. Gitzelmann, in cooperation with Th. Gerritsen, H. J. Degenhart, M. Roth and others).

XII. Hemoglobins, Porphyrins and Related Compounds: Heme Proteins (K. Winterhalter) - Porphyrins and Related Compounds (M. Doss) Bilirubin and other Bile Pigments (M. Roth).

XIII. Organic Acids in Urine Aromatic Acids (H.-Ch. Curtius,

J. A. Völlmin and H. J. Degenhart) Acids of the Tricarboxylic Acid Cycle (L. Kesner) - $\alpha$-Keto Carboxylic Acids (H.-Ch. Curtius) - Other Organic Acids (H. J. Degenhart).

XIV. Toxic Substances and Drugs (H. Brandenberger)

XV. Profeins (W. Hitzig and J. Jákó).

XVI. Tolerance Tests and Clearance Investigations (E. Leumann).

XVII. Inorganic Substances Cations (G. Ceriotti) - Anions (G. Ceriotti) - Other Metals including Heavy Metals ( $H$. Brandenberger).

XVIII. Acid-Base Bálance and Gas Analysis of Respiratory Air and Blood (R. Rispens and W. G. Zijlstra) Mass Spectrometry of Blood and Respiration Gases (A. M. Lawson) 